\title{
A continuously towed vertical bipole source for marine magnetometric resistivity surveying
}

\author{
Nobukazu Seama ${ }^{1,2}$, Noriko Tada ${ }^{2}$, Tada-nori Goto ${ }^{3}$, and Masashi Shimoizumi ${ }^{4}$ \\ ${ }^{1}$ Department of Earth and Planetary Sciences, Kobe University, Kobe 657-8501, Japan \\ ${ }^{2}$ IFREE, Japan Marine Science and Technology Center, Kanagawa 237-0061, Japan \\ ${ }^{3}$ Department of Civil and Earth Resources Engineering, Kyoto University, Kyoto 606-8501, Japan \\ ${ }^{4}$ Kyushu Polytechnic College, Kita-Kyushu 802-0985, Japan
}

(Received August 1, 2012; Revised January 11, 2013; Accepted March 12, 2013; Online published September 17, 2013)

\begin{abstract}
We propose to use an approximately vertical bipole electric current towed by a ship as a source for a Magnetometric Resistivity (MMR) method. This proposal requires the precise positioning of the bottom electrode for the bipole source, and our newly developed MMR system achieved this. We conducted an MMR experiment in the central Mariana Trough, and we obtained data using two different methods along a survey line: one method towed a bipole source transmitting continuously along the survey line, and the other used a conventional vertical bipole source transmitting at several stationary transmission stations along the survey line. We found that the towed bipole source tilted from the vertical by an angle of 8 degrees at the maximum during the MMR experiment. We compared the results from the two methods to evaluate the towed bipole source method. Our results indicate that the tilted bipole source approximates well with the vertical bipole source at the mid-point between the surface and the bottom electrodes. Since the towed bipole source method requires much less survey time and the results show a higher spatial resolution, it is a powerful tool for MMR experiments to image a shallow oceanic crustal resistivity structure efficiently.
\end{abstract}

Key words: Magnetometric Resistivity method, towed bipole electric current source, oceanic crust electrical resistivity structure.

\section{Introduction}

The Magnetometric Resistivity (MMR) method is a controlled source method and a logistically simple electromagnetic technique to investigate the shallow oceanic crust, that involves two components: a vertical bipole electric current as a source, and separate ocean bottom magnetometers as receivers. In the simplest one-dimensional model case, an approximation of the electrical resistivity of the seafloor $\rho$, is obtained from the vertical bipole electric current $I$, the amplitude of the induced horizontal magnetic field at the receiver $B$, and the source-receiver separation $R$, using the relation:

$$
\frac{B}{I}=\frac{\mu \cdot \rho_{\mathrm{o}} \cdot D}{4 \pi} \cdot \frac{1}{R^{2} \cdot \rho},
$$

where $\rho_{0}, \mu$, and $D$ are the resistivity of the ocean, the magnetic permeability in the air, and the water depth, respectively (Edwards et al., 1981). This relation indicates that the amplitude of the induced horizontal magnetic field is inversely proportional to the resistivity of the seafloor and to the square of the source-receiver separation; the larger magnetic field amplitude represents a smaller resistivity for a given separation. Nobes et al. $(1986,1992)$ conducted a MMR survey in the hydrothermally active Middle Valley off the Juan de Fuca Ridge. These were the first deep water

Copyright (C) The Society of Geomagnetism and Earth, Planetary and Space Sciences (SGEPSS); The Seismological Society of Japan; The Volcanological Society of Japan; The Geodetic Society of Japan; The Japanese Society for Planetary Sciences; TERRAPUB.

doi:10.5047/eps.2013.03.007 applications of the technique. Evans et al. (1998, 2002) determined resistivity models at depths up to $1 \mathrm{~km}$ beneath the seafloor, both at the ridge axis and further away. Resistivity models near the spreading axis generally have a lower resistivity than those in the off-axis area. Evans et al. (1998) concluded that lower axial resistivities within the uppermost $600-800 \mathrm{~m}$ of crust are associated with a recent shallow dike intrusion event and subsequent high-temperature fluid circulation at the Juan de Fuca Ridge. Evans et al. (2002) also explained the lower axial resistivities on the East Pacific Rise by the presence of hotter pore-fluids beneath the ridge crest. Tada et al. (2005) presented a one-dimensional electrical resistivity structure over a region of hydrothermal circulation in the spreading axis of the central Mariana Trough using an MMR experiment.

All the previous studies (Nobes et al., 1986, 1992; Evans et al., 1998, 2002; Tada et al., 2005) used a "vertical" bipole electric current as a source, which was transmitted from several stationary transmission stations. We call this the "stationary source method" hereafter. The reason for using a bipole electric current source (called "bipole source" hereafter) in a vertical position is that this is what the original MMR method called for, and the data analysis technique was developed for it (Edwards et al., 1981). The other reason the bipole source is vertical is because the tilt angle of the bipole source need not be measured: the measurement would be logistically complicated. Towing a bipole source by a ship is attractive for an MMR survey because the spa- 


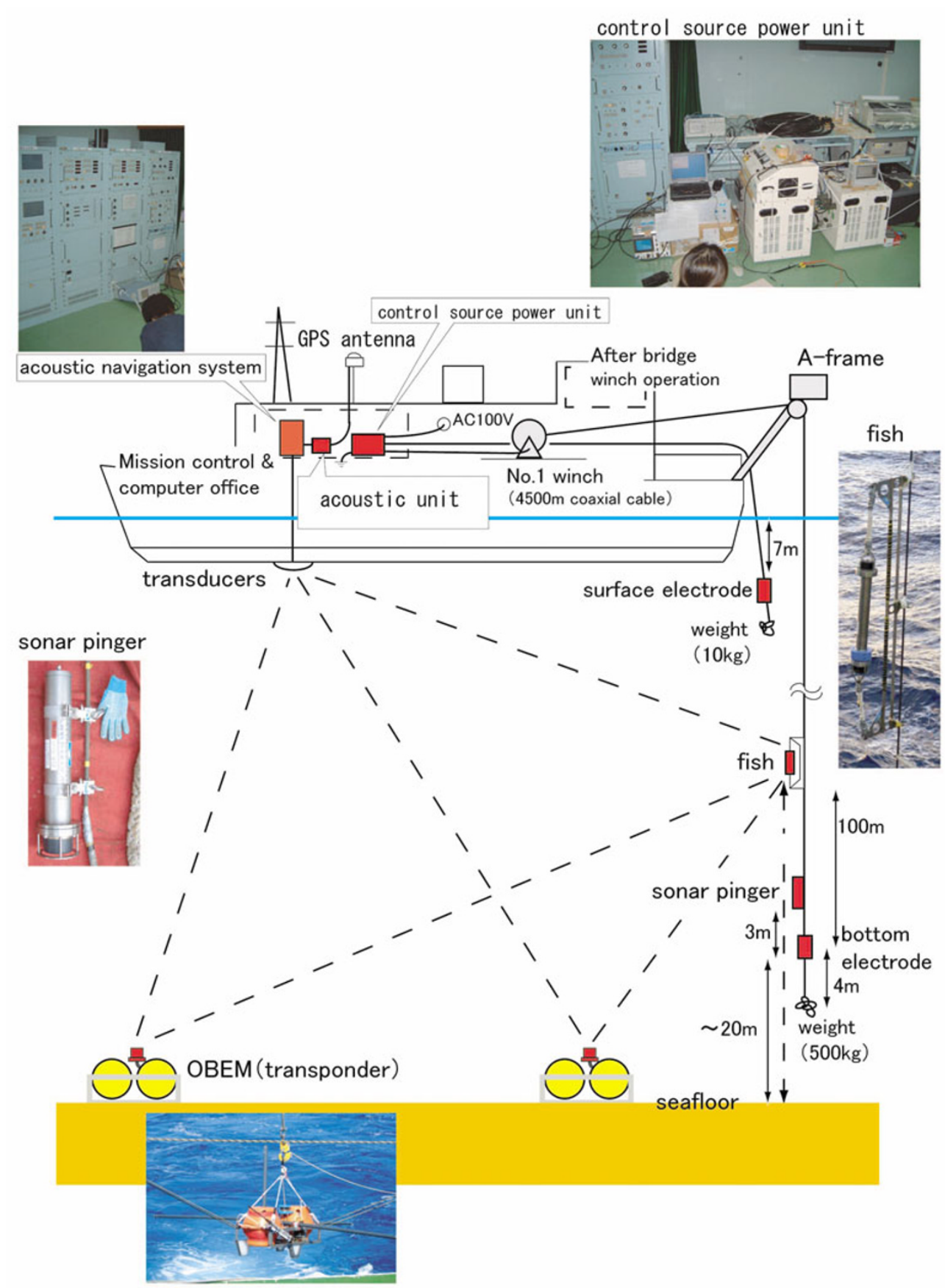

Fig. 1. Schematic features of our new MMR system.

tial coverage can be widened efficiently. But it must be remembered that the bipole source may not be vertical due to its movement through the water.

In this paper, we propose to tow a bipole electric current as a source for the MMR method (we call it the "towed source method" hereafter) and to evaluate this using a newly developed MMR system. First, we introduce our new MMR system and propose the towed source method as an application of our new MMR system. Second, we describe an MMR experiment in the central Mariana Trough and its data analysis. Then, we compare the results of the towed source method with those of the stationary source method along a survey line to evaluate the towed source method. Finally, the advantages in the towed source method are discussed.

\section{Our New MMR System and Its Application}

We have developed an MMR system, which consists of three components: equipment for a bipole electric current as a source, ocean bottom electro-magnetometers (OBEMs) as receivers, and equipment for their positioning (Fig. 1). A bipole electric current is transmitted between a surface electrode and a bottom electrode using a control source power unit. The control source power unit generated a rectangular wave-form with 5 different periods of $1,2,4$, 8 , and 16 seconds, and its peak voltage can be controlled up to \pm 240 Volts. The output current for the electrodes is monitored and its value is continuously recorded. We would expect a slight variation when the depth of the bottom electrode is changed. The electrode is made of a stainless 


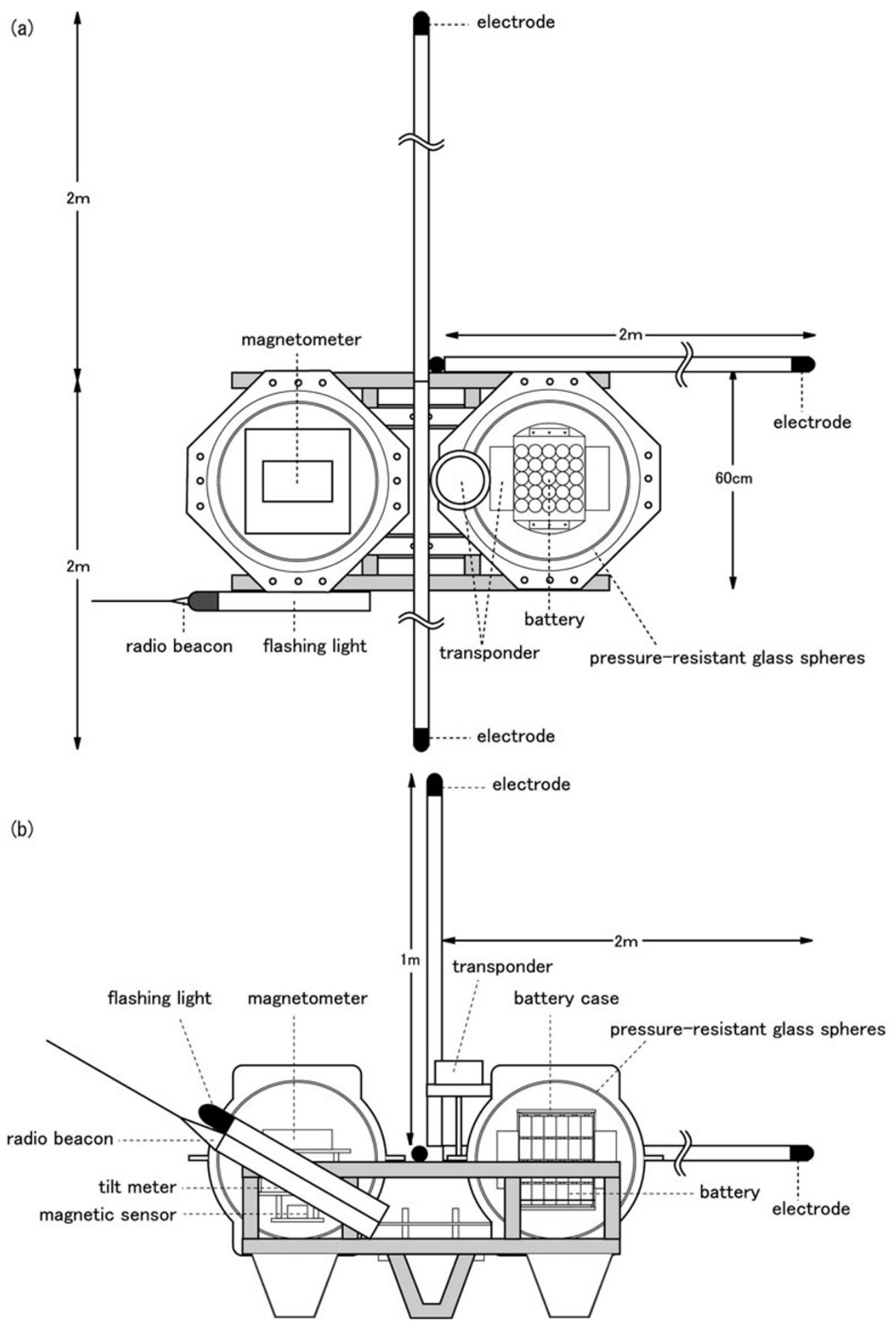

Fig. 2. Schematic features of our OBEM shown in overview (a) and in cross-section (b).

steel pipe $1 \mathrm{~m}$ in length and $14 \mathrm{~cm}$ in diameter. The surface electrode is lowered $7 \mathrm{~m}$ from the sea surface to avoid contact with the ship. The bottom electrode is connected to a separate coaxial cable within the winch wire and is lowered to just above the seafloor.

The OBEMs are used as receivers for the bipole source, and they measure three components of the magnetic field variation, three components of the electric field variation, two components of instrument tilt, and temperature. Each
OBEM (Fig. 2) has two pressure-resistant glass spheres. One contains fluxgate type magnetometers, voltmeters, and tilt meters, and the other contains battery packs and a transponder unit. It has pipes for attaching five Filloux-type silver-silver chloride electrodes (Filloux, 1987). The data sampling interval is 1 second at the minimum and this interval is used for the MMR experiment. All data are recorded internally on a flash card. A transponder is also attached to each OBEM, which enables us to determine each location. 
The OBEM also has a radio beacon and a flashing light for recovery.

Positioning the source and receivers of the MMR system is essential and several different types of equipment are used to estimate their locations. The ship position is determined by a Global Positioning System (GPS) and the ship position is simultaneously used along with other positioning equipment, since the ship position is a reference for the other positioning. The location of an OBEM is determined by an acoustically-measured distance from the ship using the transponder attached to the OBEM. Positioning the source is required to estimate the locations of both the surface and bottom electrodes. The location of the surface electrode is easily estimated from the ship location because the relative location of the surface electrode to the ship is nearly constant. To estimate the location of the bottom electrode, an acoustic unit (we call this the "fish" hereafter) is fixed to the winch wire at $100 \mathrm{~m}$ above the bottom electrode. The fish measures pressure (corresponding to water depth), altitude (using acoustic reflection from the ocean bottom), and slant ranges to the ship and also to OBEMs. An onboard acoustic unit can communicate with the fish acoustically to control it and to obtain its data in real time. A sonar pinger is also attached to the winch wire at $3 \mathrm{~m}$ above the bottom electrode to monitor its altitude from the ocean bottom in real time. The fish and the OBEM also utilize the inbuilt SSBL (super short base line) system on board R/V Kairei to provide real-time $x, y, z$ positions in the water column. The accuracy for the SSBL system is due to the angle measurement being less than 2 degrees to the object, which corresponds to $130 \mathrm{~m}$ of the positioning scatter when the object is at $3600 \mathrm{~m}$ in depth.

An important feature of our new MMR system is the precise positioning of the bottom electrode. The fish at $100 \mathrm{~m}$ above the bottom electrode can measure the distance from the fish to OBEMs, which is an important parameter in estimating the resistivity structure of the oceanic crust. Further, the sonar pinger tracks differences in height from the ocean floor of the bottom electrode, and this real time monitoring allows us to adjust the wire length to maintain a nearly constant altitude of the bottom electrode, which ensures an even coupling with the seafloor from location to location. Moreover, winch operation is available during bipole source transmission because the slip ring in the winch maintains the connection between the control source power unit and the coaxial cable in the winch wire during operation. Thus, our system allows us to tow the bipole source while maintaining a constant altitude of the electrode from the bottom through winch operation, by monitoring the altitude in real time.

We propose to use the towed bipole source for MMR experiments, as an application of our new MMR system. The bipole source is supplied continuously along a survey line and the location of the bipole source moves as the ship moves. There are two obvious advantages for the towed source method compared to the stationary source method. The first one is the time required for the survey. The towed source method is much faster and more efficient than the stationary source method. In other words, a survey using the towed source method can cover a wider area than one using the stationary source method. The second advantage is a denser spatial distribution of the source locations. Since the towed source method transmits the bipole source continuously as the ship moves, the source locations are in a line along the ship track which avoids problems seen in the stationary source method. In practical terms, the spatial distribution for the towed source method is limited by two factors: the speed of the towed source, and the resolution limitations of OBEMs to measure the amplitude of the induced magnetic fields. When the amplitude becomes too low, the stacking of longer data is required to obtain a reliable amplitude, which results in a coarser spacing of source locations.

\section{MMR Experiment and Data Analysis}

We used data from an MMR experiment near the spreading axis in the central Mariana Trough (Fig. 3) during the R/V Kairei KR02-14 cruise (Seama et al., 2003) to evaluate the towed source method. Both the towed source and the stationary source methods were applied along a survey line (the white line in Fig. 3) during the MMR experiment. For the evaluation, we used induced magnetic field data obtained from one OBEM (OBEM2 in Fig. 3) as the receiver, together with acoustic ranging data between the fish and each OBEM. The OBEM also measured the induced electric field, but we do not use this data in this paper. It is available should it be needed in the future.

The bipole source, which generated a rectangular waveform with a period of 16 seconds, with its peak current at approximately $\pm 19 \mathrm{~A}$, and its peak voltage at \pm 170 Volts, was applied between the surface electrode and the bottom electrode on the end of the winch wire just above the seafloor. The bipole source was supplied continuously along the survey line (a white line in Fig. 3) for the towed source method, while it was supplied at 10 transmission stations (dots in Fig. 3) along the survey line for the stationary source method. The towed bipole source moved at a speed of approximately $1 \mathrm{~km} /$ hour following the ship, and the towed bipole source may not have been vertical due to its movement. For the stationary source method, the vertical bipole source was supplied at each transmission station. In this second pass, the ship was kept on each transmission station for 30 minutes to allow stacking at the periodic wave signals, improving the signal to noise ratio. On completion of a station, the wire was rewound about $100 \mathrm{~m}$, and the ship moved to the next site. Once on a new station, the wire was set as vertically as possible before relowering it to the seafloor, so that the source was kept as a vertical bipole.

The survey times (Seama et al., 2003) indicate that the towed source method was nearly twice as efficient as the stationary source method. Except for the common time for lowering and raising of the winch line between the sea surface and the ocean bottom (4.5 hours), the actual survey time for the towed source method along the survey line was 4 hours, while this was 9.5 hours for the stationary source method with 10 stationary sources. Even though there was a big difference in the actual survey time, the transmission times were more similar; the towed source method used all the actual survey time solely for transmitting electric current (4 hours), while the stationary source method used only 


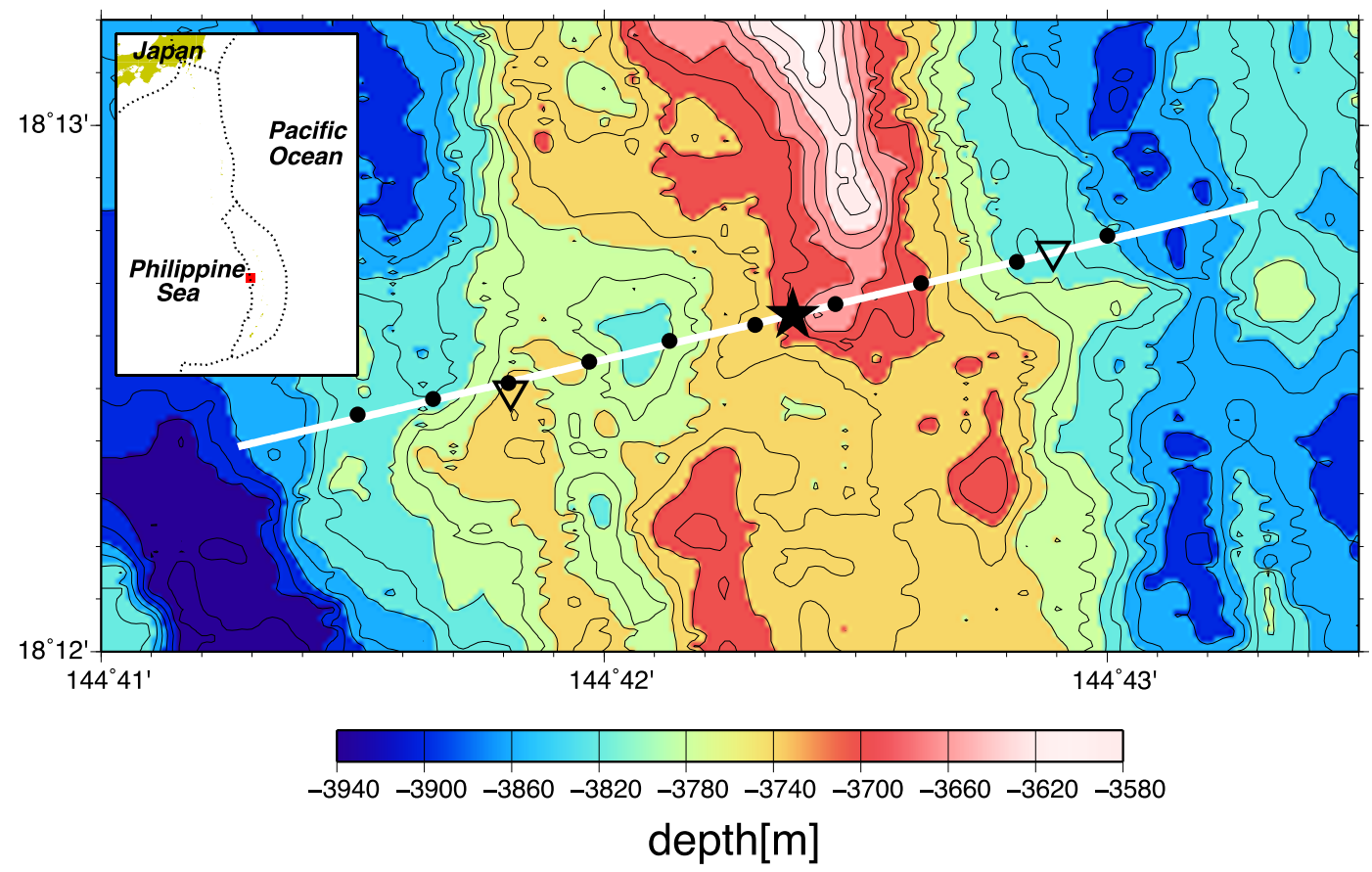

Fig. 3. Bathymetry map of our MMR experiment in the central Mariana Trough with contour intervals of $20 \mathrm{~m}$. Inset shows a regional map of the MMR experiment location (solid square) with plate boundaries (dotted line). Locations of the receiver (OBEM2), acoustic bases (OBEM1 and OBEM3), and 10 transmission stations are shown by a star, triangles, and dots, respectively. A white line denotes the survey line.

half of the time for transmitting electric current ( 5 hours) and the other half was used for moving the ship between transmission stations (4.5 hours). So overall, the towed source method took half the time needed by the stationary source method, showing nearly double the efficiency.

We used the relation between the source-receiver separation and the amplitude of the induced magnetic field at the receiver to evaluate the towed source method, because this relation is fundamental information needed to estimate the electrical resistivity of the seafloor as described in the Introduction. The magnetic field generated by the bipole source is inversely proportional to the resistivity of the seafloor and to the square of the source-receiver separation; the larger magnetic field amplitude represents a smaller resistivity for a given separation. Calculations of the source-receiver separation and the amplitude of the induced magnetic field derive the relation. A one-dimensional resistivity structure model using the relation of the stationary source method has been derived by Tada et al. (2005). We will compare the relation for the towed source method with that for the stationary source method along the survey line. The comparison allows us to evaluate the towed source method. We expected that both relations would be similar because the survey line is the same. However, we have some reservations because the bipole source of the towed source method was probably not vertical.

Amplitudes of the horizontal magnetic field obtained by the stationary source method were calculated in the same manner of Tada et al. (2005), which is as follows. First, we chose time series data sets of three-component magnetic field data since the transmission of the electric current would be 30 minutes each. Second, each data set was divided into 13 segments with lengths of $256 \mathrm{sec}-$ onds; the segments overlapped by half their length (128 seconds) to obtain as many segments as possible. Third, the power spectrum was estimated by calculating the fast Fourier transform for each data segment. The amplitude of each data segment was examined by comparing this power spectrum with one from a reference rectangular wave with $1 \mathrm{nT}$ amplitude and a period of 16 seconds. Fourth, the horizontal component of the magnetic field variation was separated from the vector magnetic field using the OBEM attitude based on the tiltmeter measurement. Fifth, the horizontal component of the magnetic field was normalized by the current intensity of the bipole source. Finally, the average and the standard deviation of the amplitude at each site were estimated from the horizontal magnetic field data of the 13 segments.

Amplitudes of the horizontal magnetic field obtained by the towed source method were calculated from the threecomponent magnetic field data in a similar manner to that of the stationary source method. A major difference in the calculation for the towed source method is that only one segment with its length of 256 seconds is used and that the resultant value becomes one data point of the amplitude at the mid-point of the time in the segment. The reason for this calculation is that the amplitude varies with time as the towed source moves. The amplitude obtained using this calculation is a value averaged over distance as the towed source moves for 256 seconds; this corresponds to $70 \mathrm{~m}$ when the speed of the towed source movement is $1 \mathrm{~km} /$ hour. The next segment for the calculation overlaps the previous one by one half of its length (128 seconds), and the manner of the calculation is the same as that of the stationary source method.

The source-receiver separation is defined as the horizon- 


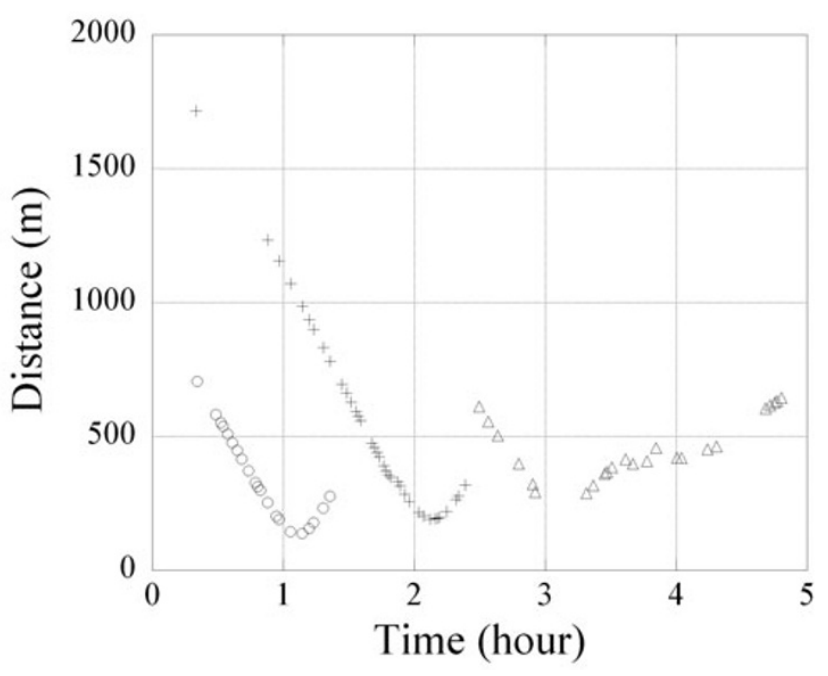

Fig. 4. Distances from the fish to OBEM1 (circles), OBEM2 (crosses), or OBEM3 (triangles), shown against time.

tal distance between the source and the receiver. This can be calculated when the location of the source and the receiver is known. The location of the receiver (OBEM2) was estimated from distances between OBEM2 and the ship, which were acoustically measured at several different ship locations; the ship locations are simultaneously determined by GPS. For the stationary source method, the source location is the transmission station because the bipole source is vertical. The transmission station is equivalent to the ship location determined by GPS. Then, the source-receiver separation was calculated from the ship location (source) and the OBEM2 location (receiver) for the stationary source method.

The source-receiver separation for the towed source method has a range because the source is not vertical; the location of the source has a range due to the difference in horizontal locations between the surface and the bottom electrodes of the bipole source. Then, the source-receiver separation for the towed source method can be derived from two end members. The first one is the horizontal distance between the receiver and the surface electrode of the bipole source, and this distance is the same as that in the stationary source method. The second one is the horizontal distance between the receiver and the bottom electrode of the bipole source. This horizontal distance is calculated from an acoustic measurement of the distance from the fish attached to the winch wire at $100 \mathrm{~m}$ above the bottom electrode to three OBEMs along the survey line. Figure 4 shows the distances between the fish and each OBEM obtained from the acoustic measurement over time. 21, 37, and 24 data points were obtained for OBEM1, OBEM2, and OBEM3, respectively; the measurement was made continuously, but some measurements failed. The horizontal distance between the fish and each OBEM was derived by assuming the fish moved along a line with a constant offset from each OBEM. The offset was composed of two components; one was the difference in depth between the fish and each OBEM, and the other was the horizontal distance perpendicular to the survey line between the fish and each OBEM. The offset from each OBEM was estimated as the closest distance to each OBEM from the fish along the survey line using the acoustic measurement data (Fig. 4). Then, the horizontal distance between each OBEM and the fish was calculated. Finally, the horizontal distance between the receiver (OBEM2) and the bottom electrode (fish) was calculated using horizontal distances between the OBEMs. Each distance at the time when the amplitude of the induced magnetic field was obtained at the receiver was calculated using interpolation within the acoustic measurement (from -1715 to $1550 \mathrm{~m}$; positive distance is toward the east from the receiver along the survey line) or extrapolation outside this region.

\section{Results and Discussion}

The horizontal distances from the receiver (OBEM2) to the ship and to the fish are shown in Fig. 5(a). Positive distance is east of the receiver along the survey line and the distances correspond to the locations of the ship and of the fish along the survey line from the origin of the receiver. This estimation of the fish location can be evaluated by a comparison of two bathymetric data, because they should match when their locations are the same. One set of bathymetric data was obtained from the R/V Kairei multi-narrow beam of a SeaBeam 2112, and the other set of bathymetric data was from the sum of depth and altitude data obtained from the fish (Fig. 6). These two bathymetric sets of data match well in general, indicating that the fish location is well estimated using our new MMR system. Some scatter in the bathymetric data from the fish is due to the scatter of the altimeter measurements.

The horizontal distance between the ship and the fish is calculated from the locations of the ship and of the fish (Fig. 5(b)). This horizontal distance varies up to $500 \mathrm{~m}$, which corresponds to 8 degrees of the bipole source tilt from the vertical (Fig. 6). The horizontal distance does not show any correlation to small changes in the ship speed, but large changes in the ship speed with some time lag show a good correlation (Fig. 5(c)); the horizontal distance becomes much smaller when the ship slows down, but it takes time for the horizontal distance to become a minimum even when the ship speed is nearly zero.

The source-receiver separation for the towed source method is based on a range of measurements due to differences in the horizontal locations between the surface and the bottom electrodes of the bipole source. The amplitudes of the induced magnetic fields are plotted against three different horizontal distances: from the receiver to the surface electrode, to the bottom electrode, and to the mid-point between the surface and the bottom electrodes (Fig. 7). The results indicate that the relation for the towed source method between the source-receiver separation and the amplitude coincides with that for the stationary source method when the horizontal distance from the receiver to the mid-point between the surface and the bottom electrodes is chosen as the source-receiver separation for the towed source method (Fig. 7(c)). The other two choices result in large differences between the towed source and the stationary source methods (Figs. 7(a) and 7(b)). Thus, our results indicate that the tilted bipole source approximates well with the vertical 
(a)

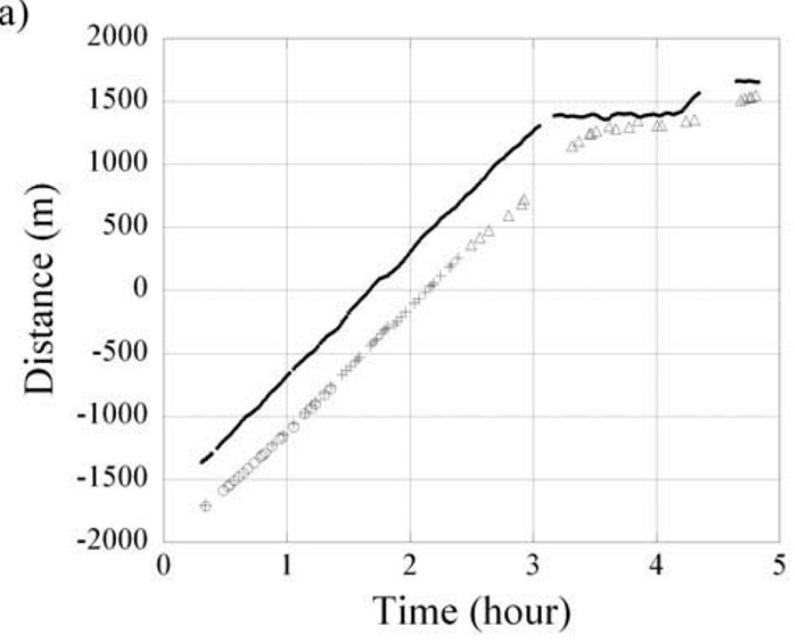

(b)

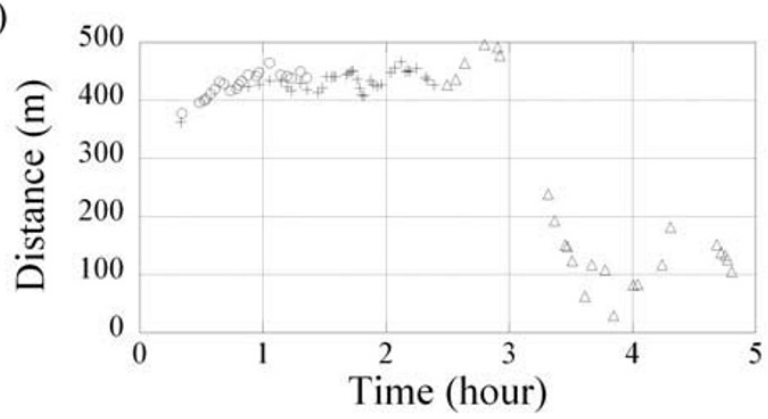

(c)

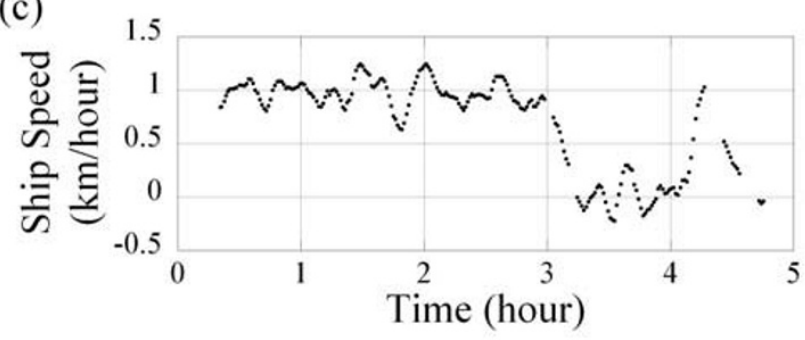

Fig. 5. (a) Horizontal distances of the ship (dots) and the fish (the other symbols) from the receiver (OBEM2) along the survey line (positive distance is toward the east from the receiver along the survey line), (b) horizontal distance between the ship and the fish, and (c) ship speed, are all plotted against time. Each symbol in (a) and (b) indicates its data source; distance measurement from the fish to OBEM1 (circles), OBEM2 (crosses), or OBEM3 (triangles), as shown in Fig. 4. It is worth noting that the horizontal distance between the ship and the fish is in proportion to the tilt angle of the towed bipole source when the tilt angle is small, and that a tilt angle of 1 degree corresponds to $63 \mathrm{~m}$ in the horizontal distance between the ship and the fish in this case.

bipole source at the mid-point between the surface and the bottom electrodes. The accuracy of this approximation can be confirmed using analytical or numerical methods in the future, but the present approximation is useful for practical modeling; at least in the case when the bipole source tilt angle is less than 8 degrees from the vertical, as in the present case.

The results from the towed source method show a denser spatial distribution of the source locations than those from the stationary source method, even though the survey times indicate that the towed source method is nearly twice as

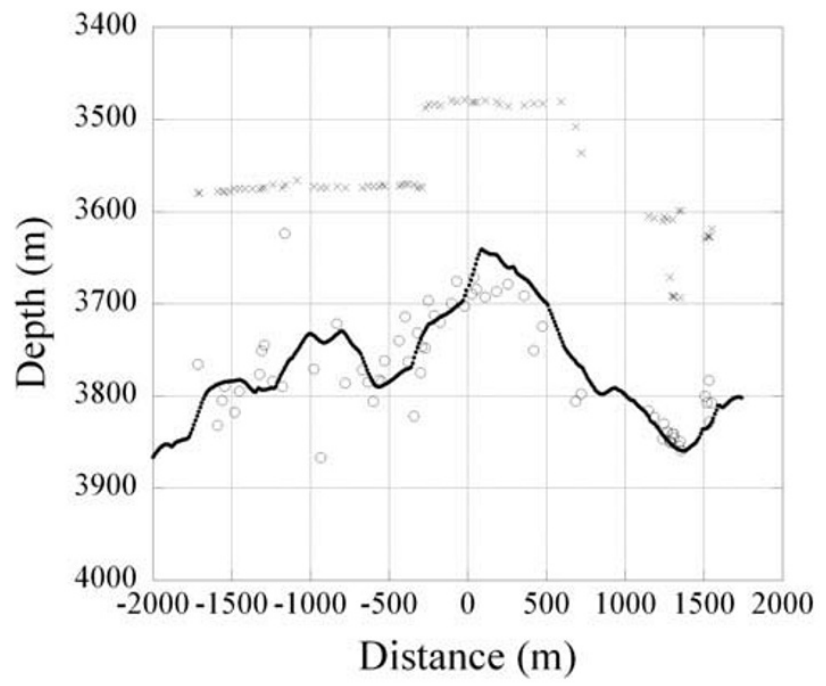

Fig. 6. Depth of the fish (crosses) and bathymetry are shown against the horizontal distance of the fish from the receiver along the survey line (positive distance is toward the east from the receiver along the survey line). The bathymetric data were obtained from the R/V Kairei multi-narrow beam of a SeaBeam 2112 (dots), and from the sum of the depth and altitude of the fish (open circles).

efficient as the stationary source method as described in Section 3. The denser spatial distribution shows an outline of changes in the relation between the source-receiver separation and the amplitude of the induced magnetic field at the receiver (Fig. 7(c)), and clarifies the following two points. First, an anomalous amplitude feature in the relation is clearly identified (Fig. 7(c)). The relation shows that amplitude decreases as the source-receiver separation increases in general. We found an outlier from the trend with a higher amplitude at a distance of $-750 \mathrm{~m}$ in the results from the stationary source method. The results from the towed source method clarified that this anomaly occurs only in a narrow area within a width of $100 \mathrm{~m}$. The higher amplitude feature suggests a narrow local low resistivity area, where electric current penetrates deeper to induce the higher amplitude of the magnetic field. Second, the asymmetric feature of the relation is also clearly identified (Fig. 8). Magnetic field amplitude induced by the bipole source to the east side of the receiver is clearly higher than that of the west side, suggesting a lower resistivity structure to the east. These features are important in the estimation of two-dimensional resistivity structures in the future, although a modeling code is required for this purpose.

Our results also indicate two cautions when using the towed source method. First, an anomalous low amplitude is recognized between 200-300 m (Fig. 7(c)). Since this feature occurs when the bottom electrode passes over the receiver (zero horizontal distance between the bottom electrode and the receiver (Fig. 7(b))), our method to obtain the amplitude of the induced magnetic field at this point remains problematic. The problem pertains to the fact that each component of the induced magnetic field varies so rapidly at this point that our data analysis window of 256 seconds cannot cope. Another possible cause of the anomalous low amplitude is that it is due to the effect of the towed 
(a)

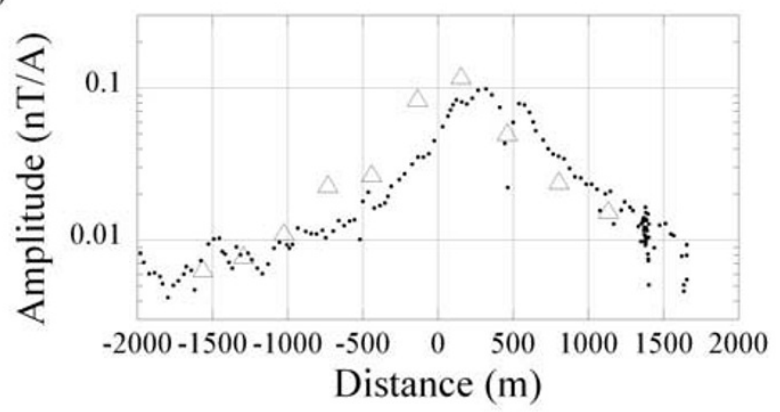

(b)

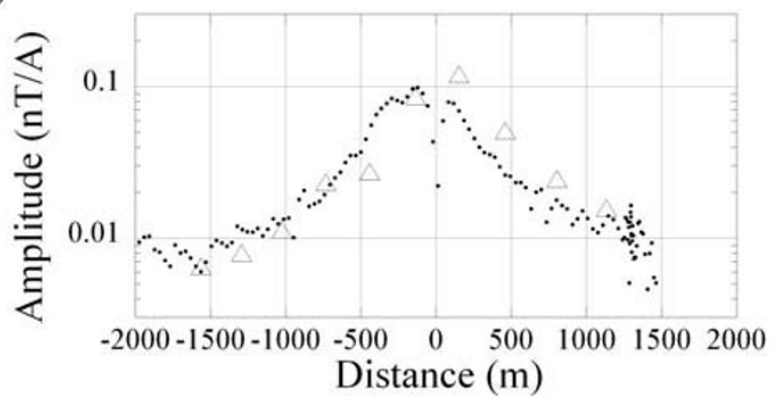

(c)

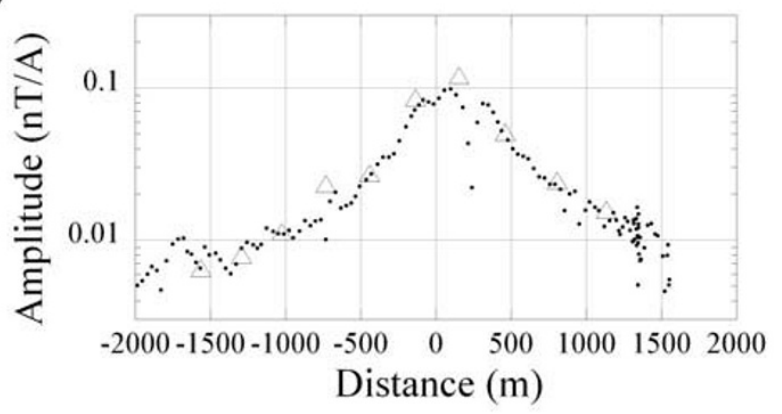

Fig. 7. Relation between the horizontal distance of the source from the receiver (positive distance is toward the east from the receiver along the survey line) and the amplitude of the induced magnetic field recorded by the receiver. A comparison of both the stationary source method (triangles) and the towed source method (dots) are shown. Three different horizontal distances for the towed source method were calculated due to three different locations of the bipole source: the surface electrode (a), the bottom electrode (b), and an estimation of the mid-point between the surface electrode and the bottom electrode (the mid-point of the bipole source) (c).

source tilt being large near the bottom electrode. The second point is that we found a decrease, and not an increase, in the amplitude as expected in a $100 \mathrm{~m}$ range when the source-receiver separation was near zero (Fig. 7(c)). This is probably because the source-receiver separation is so close that our approximation results are not valid.

\section{Conclusion}

Our newly developed MMR system allows us to tow a nearly vertical bipole electric current source. The bottom electrode can be controlled at a constant altitude from the ocean bottom through winch operation by monitoring the altitude in real time. Precise positioning of the bottom electrode in the system determines the horizontal distance between the surface electrode and the bottom electrode. The

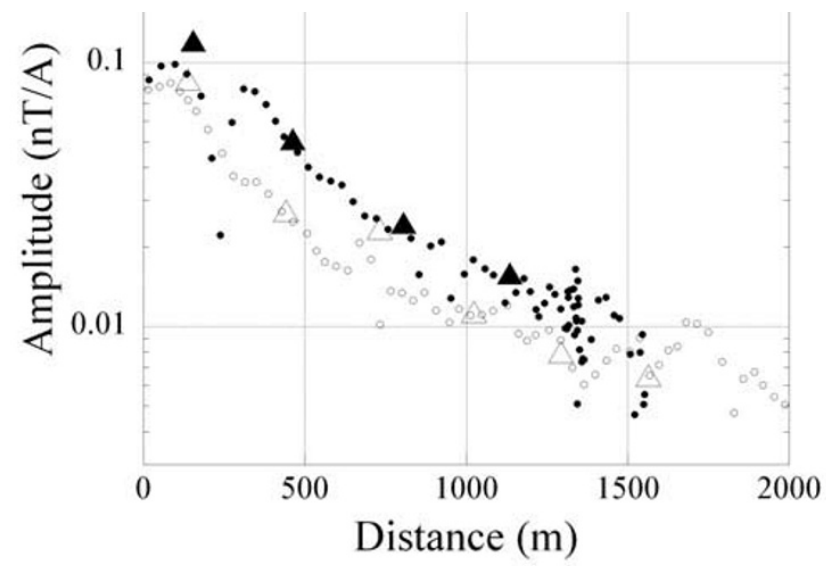

Fig. 8. The relations between the source-receiver horizontal distance and the amplitude of induced magnetic field for the stationary source method (triangles) and for the towed source method (circles) are shown. Open and solid symbols show the sources that locate to the west and to the east of the receiver, respectively. The mid-point between the surface and the bottom electrodes is chosen as the bipole source location for the towed source method to calculate the source-receiver separation shown in Fig. 7(c).

horizontal distance shows that the towed bipole source was tilted from the vertical by an angle of no more than 8 degrees during the MMR experiment in the central Mariana Trough. Our results indicate that the tilted bipole source approximates well with the vertical bipole source at the midpoint between the surface and the bottom electrodes. The survey times of both methods indicate that the towed source method has nearly double the efficiency of the stationary source method. Furthermore, the results from the towed source method show a higher spatial resolution because the spatial distribution of the source locations is denser in the relation between the source-receiver separation and the amplitude of the induced magnetic field. We conclude that the towed bipole electric current source is a powerful tool for MMR experiments to image efficiently a shallow oceanic crustal resistivity structure and to survey hydrothermal circulation systems and/or mineral deposits, as practical applications.

Acknowledgments. We would like to thank the scientific members, the captain, and the crew of the KR02-14 cruise (R/V Kairei, JAMSTEC) for their help. This work is partly supported by the scientific program of "TAIGA" (Trans-crustal Advection and Insitu reaction of Global sub-seafloor Aquifer)" sponsored by the MEXT of Japan. We would also like to thank Taichi Kawakami, Shigeo Matsuda, and Yoshinori Azuma for their help developing our new MMR system. Kerry Key and one anonymous reviewer provided helpful comments. Kumiko Okumura drew some figures and helped to prepare the manuscript. Donald Moore read the manuscript and offered helpful improvements. GMT software (Wessel and Smith, 1998) was used to create the bathymetry map of the survey area.

\section{References}

Edwards, R. N., L. K. Law, and J. M. DeLaurier, On measuring the electrical conductivity of the oceanic crust by a modified magnetometric resistivity method, J. Geophys. Res., 86, 11,609-11,615, 1981.

Evans, R. L., S. C. Webb, M. Jegen, and K. Sananikone, Hydrothermal circulation at the Cleft-Vance overlapping spreading center: Results of a magnetometric resistivity survey, J. Geophys. Res., 103, 12,321-12,338, 
1998.

Evans, R. L., S. C. Webb, and the RIFT-UMC Team, Crustal resistivity structure at $9^{\circ} 50^{\prime} \mathrm{N}$ on the East Pacific Rise: Results of an electromagnetic survey, Geophys. Res. Lett., 29, doi:10.1029/2001GL014106, 2002.

Filloux, J. H., Instrumentation and experimental methods for oceanic studies, in Geomagnetism Vol. 1, edited by J. A. Jacobs, pp. 143-248, Academic Press Inc., Florida, 1987.

Nobes, D. C., L. K. Law, and R. N. Edwards, The determination of resistivity and porosity of the sediment and fractured basalt layers near the Juan de Fuca Ridge, Geophys. J. R. Astron. Soc., 86, 289-317, 1986.

Nobes, D. C., L. K. Law, and R. N. Edwards, Results of a sea-floor electromagnetic survey over a sedimented hydrothermal area on the Juan de Fuca Ridge, Geophys. J. Int., 110, 333-346, 1992.

Seama, N., T. Goto, Y. Nogi, M. Ichiki, T. Kasaya, N. Tada, H. Iwamoto,
K. Kitada, T. Matsuno, S. Yoshida, Y. Kawada, M. Ito, R. Ishii, K. Takizawa, K. Suyehiro, H. Utada, and M. Shimoizumi, Preliminary Report of KR02-14 Kairei cruise, JAMSTEC J. Deep Sea Res., 22, 89106, 2003.

Tada, N., N. Seama, T. Goto, and M. Kido, 1-D resistivity structures of the oceanic crust around the hydrothermal circulation system in the central Mariana Trough using Magnetometric Resistivity method, Earth Planets Space, 57, 673-677, 2005.

Wessel, P. and W. H. Smith, New, improved version of the Generic Mapping Tools released, Eos Trans. AGU, 79, 579, 1998.

N. Seama (e-mail: seama@kobe-u.ac.jp), N. Tada, T. Goto, and M. Shimoizumi 